\title{
Tissue Paper Roll Mounting for an Automatic Wet Tissue Pouch Packaging Machine
}

\author{
V. N.Chougule, A. S. Bhandari, S. S. Agrawal, K. M. Khandelwal \\ (Department of Mechanical Engineering, M. E. S. College of Engineering, S. P. Pune University, India)
}

\begin{abstract}
This paper deals with the mechanisms used in wet tissue pouch packing machine for reel mounting process. This mainly includes the splicing mechanism and use of adhesives for achieving zero downtime in order to reduce the time required for reel change, calculation of tearing force of the tissue using a spring balance, the selection of a spool holder on which the roll is placed, overcoming the fluctuations caused due to noneccentricity of roll and brake considerations for maintaining proper tension taking into account the changing diameter of feed roll with respect to time and use of sensors for job presence. It also includes calculation of a suitable motor input speed considering the variation in the diameter of the roll. The installation of accumulator to supply required tissue at a constant rate is also explained below.
\end{abstract}

Keywords - Accumulator, dancer roller (intermediate rollers), film tension, web handling, splicing.

\section{INTRODUCTION}

Roll mounting though seems to be a very simple concept of a roll being mounted onto a shaft and allowed to rotate freely, however it is not so, roll mounting is a very critical topic it consists of various considerations and aspects to be considered to feed the roll properly to the machine. Roll mounting is the primary and the most crucial part of any machine which deals with film handling, e.g. newspaper printing press, milk packaging machine, candy packaging machines, etc. Important factors to be taken into account while designing the mounts are film tension while feed, eccentric weight of the roll, rate of feed depending on the diameter of the roll, perpendicularity alignment of the roll with respect to the machine, job presence detection, achieving minimum down time and reverse torque to be applied. All these factors have to be tackled in order to achieve precision and for the whole machine to function smoothly. 11 the mechanisms for this machine have to be selected in such a way that their economic running should be given the first preference over high accuracy even though accuracy is a very important criteria too which just cannot be compromised. Thus all the mechanisms and parts selected have to be easily available locally, should have low maintenance and should be able to provide the required accuracy while working. The key components of this roll mount consists of a cantilever shaft, a plate type brake, two positioning plates, a spool holder, a suitable motor, a proximity sensor, load cell, intermediate rollers and pulley and an ultrasonic sensor. The roll had to be in perfect alignment with the intake of the machine, and it should always be concentric with respect to the shaft. For this purpose some options were available which included using feed rails, alignment plates. Tor maintaining the concentric, central spool holder was used [1].Tension is the most important parameter to be controlled during the feed of the roll. Various methods were used to control the tension which included the dancer rolls and idler weight pulley. The measurement of tension during roll feed is also very important for which use of roll cell is recommended [2]. The use of an accumulator helps to control the rate of feed of tissue as per requirement [3]. A break is a very crucial component of the mounting assembly and it is used to control and stop the shaft in order to maintain proper feed of the tissues. The use of flat plate type of break is recommended for this particular application [4].

\section{MACHINE LAYOUT AND COMPONENTS}

Any roll mounting setup consists of these basic parts which include the roll, the spool on which the roll is mounted, the spool holder on which the spool is mounted, the shaft which is used to mount the spool holder, the shaft is connected to a suitable driving motor via a suitable coupling, a brake and intermediate rollers. The roll mount also uses two key sensors namely, proximity and a load cell. The intermediate rollers are free to move vertically between certain limits, the reason and working shall be explained subsequently. Proximity sensors mounted on the intermediate rollers shall be used to indicate the extreme positions. And the load cell is used to sense the tension in the film. The intermediate rollers consist of two wheels that hold the tissue between them. 


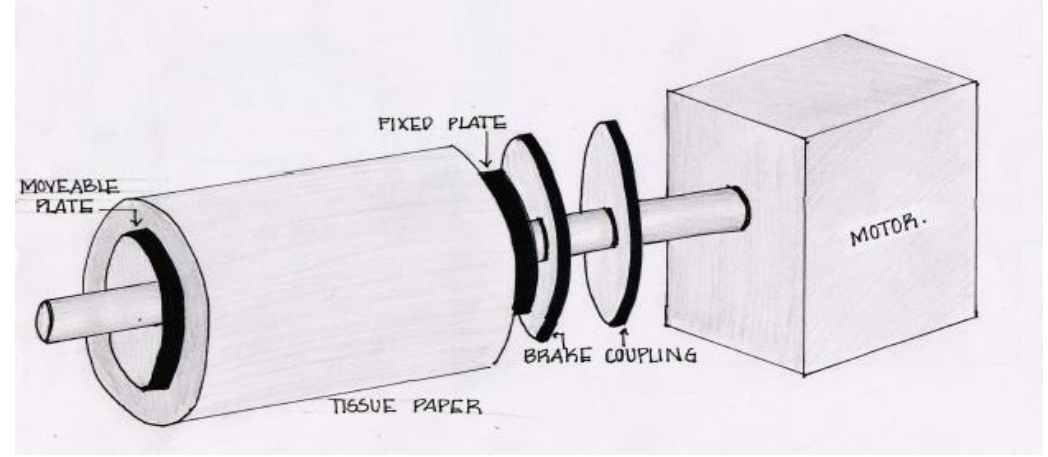

Fig.1.Assembly of shaft

3.1. Tension in the tissue film

\section{DESIGN CONSIDERATIONS}

The tension in the tissue while being fed is the most important consideration while designing the whole mounting system. A minimum constant tension has to be maintained in the film while feeding in order to ensure proper feeding of the roll, to avoid wrinkling of the tissue and also to prevent sagging.

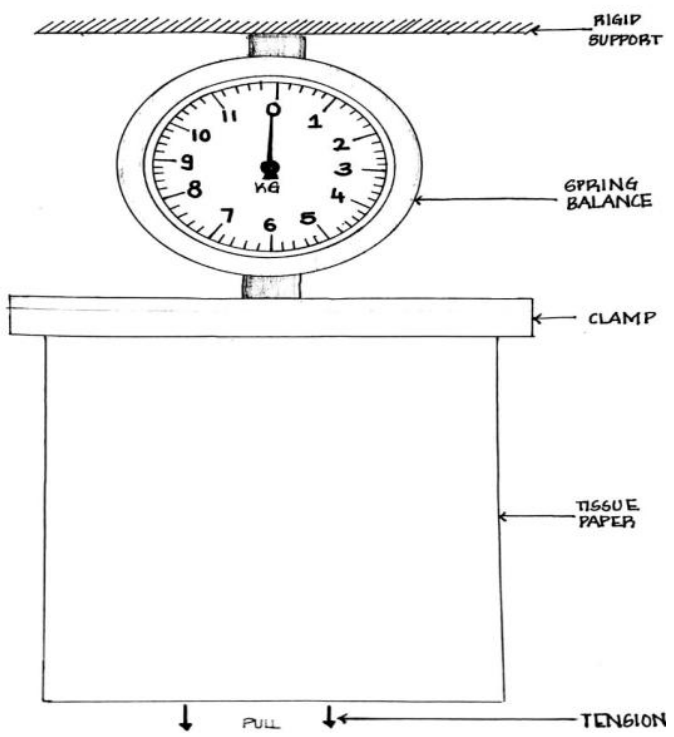

Fig.2. Spring balace for measuring tearing tension

The value of tension to be maintained should be just high enough to prevent any sagging and formation of wrinkles. While selecting the value of this tension we should be extremely careful that the tension should be low in terms that it should not cause stretching of the tissue and cause any deformation in the form of elongation. In order to determine the suitable tension a simple experiment can be carried out using a spring balance with a clamp at its end. The tissue sample clamped onto the spring balance and tension is applied in the downward direction and the tissue is observed carefully until it tears. A number of observations are carried out with varying tissue lengths and then an average of all the values at which the tissue tears is calculated. The value of tension selected to be maintained in the tissue is usually taken as one third or less of the average tearing tension found out above. The selected tension is also verified such that it should not cause any permanent elongation of the tissue when applied.

3.2 Achieving minimum downtime. Downtime refers to the time for which the machine remains stalling.

Downtime is extremely undesirable for any industry. Loss of time means loss of money. Also when the machine is stalling it is actually on but not doing any work thus consuming power which is not used, also stalling leads to overheating of the heaters used in the sealing section.In the mounting section downtime can occurs during the changing of the roll when the previous roll gets over. 


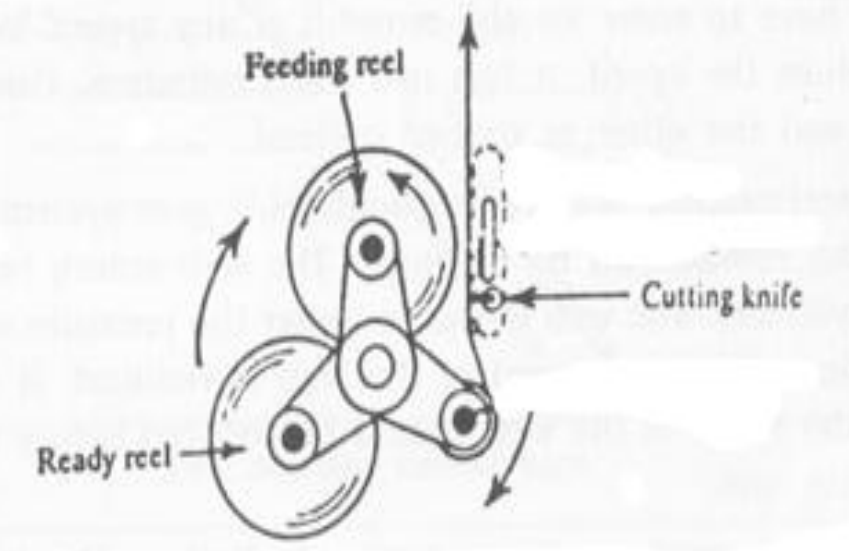

Fig.3.Splicing mechanism

There is a remedy to completely avoid downtime, i.e., by using a secondary shaft along with a splicing mechanism. Splicing basically refers to attaching one roll onto another roll using a suitable adhesive. The adhesives used may be either some form of liquid adhesive, a simple adhesive tape or a double side tape. Here we are going to use a double side tape. Some important considerations while selecting the adhesive are:

3.2.1. Easy to apply and quick in action.2.2.2. It should not react with the chemicals that are to be applied to the tissue while it passes through the machine.

3.2.3. Its bond strength should be enough so that it would be able to sustain the applied tension and not separate under it.

3.2.4. In case of tapes they should have minimum thickness so that they easily pass through the rollers.

3.3. Alignment

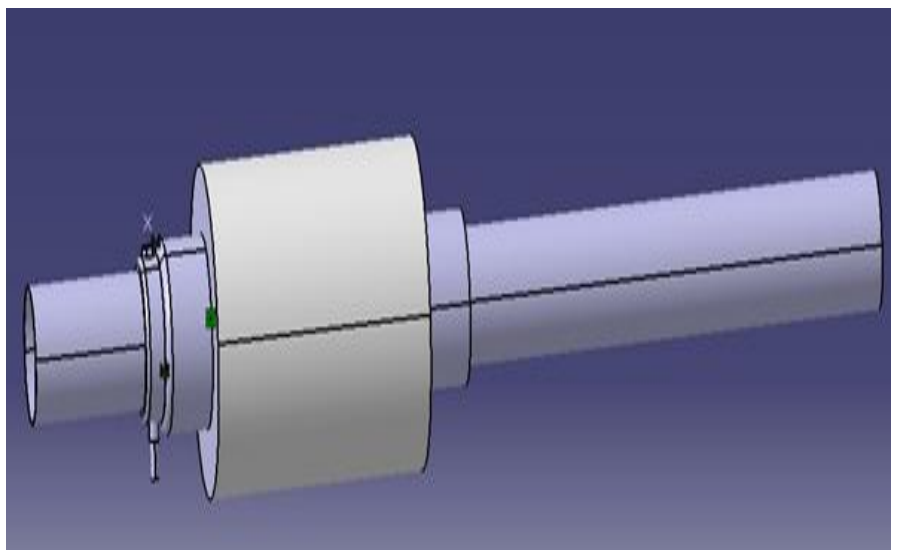

Fig.4.Roll mounting

The roll should always be held such that its axis perpendicular to the direction of feed, i.e., to the intake rollers. Special care should be taken while mounting it so that it does not lose its perpendicularity with respect to the intake rollers else it will result in jerks while feeding and also cause twisting of the roll to some extent. In order to maintain proper alignment the roll is mounted two flat plates, one being a fixed plate and the other being an adjustable plate which is screwed onto the shaft such that it tightly secures the roll on the shaft, maintains its perpendicularity and also prevents any relative motion between the shaft and the roll. The movable plate might be either clamped on the shaft or screwed using a nut and bolt.

\subsection{Variation of internal diameter of the spool}

This is a very common problem faced during roll handling in any industry though it might not be necessary always. Most of the times the internal diameter of the spool received might show a slight variation 
from one roll to another. On examining a group of 50 rolls we found out that there was a variation in the internal diameters of the spools was $\pm 1 \mathrm{~mm}$. Due to different diameters if the shafts there might often be problems while mounting the roll, if the diameter is more than the specified diameter than the spool will be loosely mounted on the shaft and there will be play in between them and if the diameter is too small then the spool will be difficult to mount and might also get damaged also damaging the shaft.

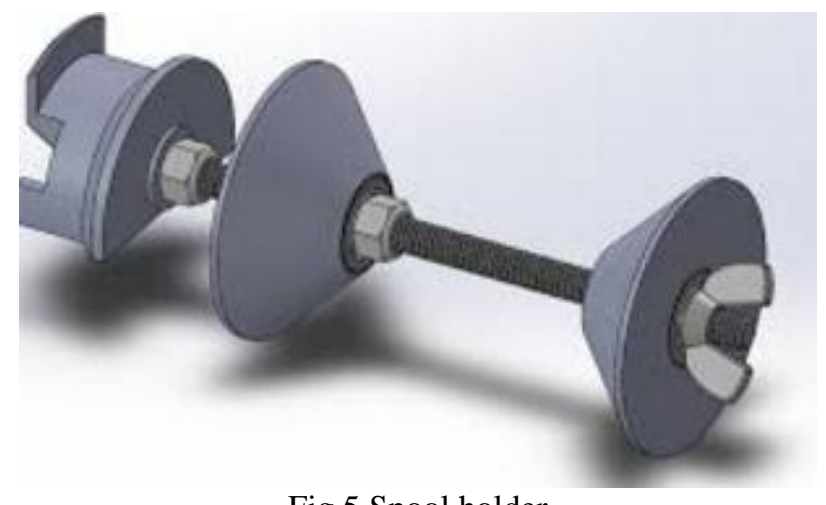

Fig.5.Spool holder

In order to mount the roll properly on the shaft we have to use a spool holder. The spool holder consists of two cones on either sideand a hollow shaft, the spool holder cone can accommodate the variation of the Internal diameter of the roll without causing any of the above problems. Spool holders are mostly purchased directly from the markets from a range of standards available.

\subsection{Web handling}

Web handling apparatus pulls web from a supported roll at a designated speed. A brake is provided for controlling the roll speed and a load cell senses changes in the tension of the web caused by changes in the pulling force of the web. The brake is controlled in accordance with the output of the load cell so that the web is maintained substantially under constant tension.

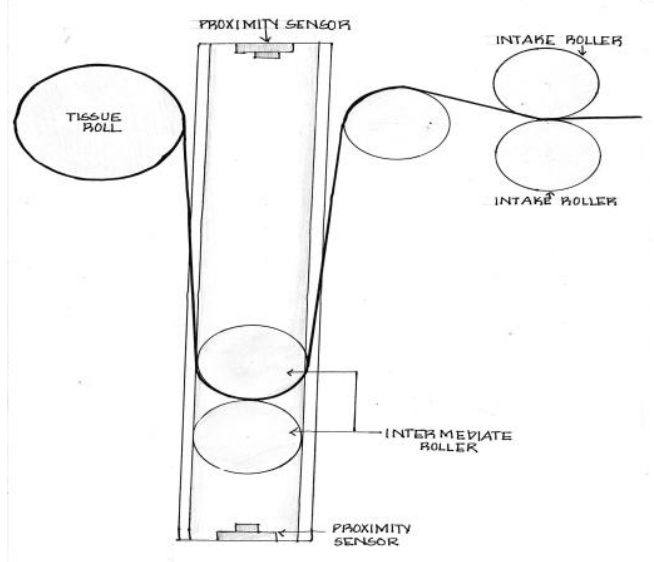

Fig.6.Accumulator

The mechanism to be chosen must provide web uninterruptedly to machines which consume the web at high speed,- a printing press, for example. The leading end of the roll of ready web is spliced to the trailing end of the depleted roll of rotating web and to deliver the web continuously in a controlled fashion to the webconsuming machine. To achieve this, the apparatus consists of an accumulator situated between the splicer and the web-consuming machine. When the rotating web is stopped to splice its trailing end to the leading edge of the ready web, there is enough material in the accumulator to supply the requirements of the web-consuming machine until the ready web roll is accelerated to running speed after the splice is made.

\subsection{Braking}

A major consideration in web handling line is the ability to properly unwind the incoming rolls of material. For proper maintenance of web tension a braking system is used which is connected to the cantilever 6th National Conference RDME 2017, 17th- 18th March 2017. 
shaft on which the roll is placed. This braking system is designed to impart the required tension to the web reel by generating the required force on the rotating roll of material. The braking system's force must stay in balance with the web force in order to maintain web tension. This is an important consideration when diameter of the roll of material continues to decrease as the material is unwound. Another really important problem face during roll feed is non-eccentric loading, i.e., the weight of the roll might not be well balanced about the centre of gravity of the roll. There might be various reasons causing this misalignment of load about the geometrical axis of the roll including improper winding of the roll, presence of folds in between different layers, defects in the spool, any damage caused to the roll while transportation and also is the roll has not been mounted on the spool holder properly. The main effect of this non-eccentric loading is that due to misbalance of the load the motion of the roll becomes jerky and also results in slackness or excess tension. This jerky motion of the roll is overcome using a suitable braking system. In our case we shall be using a circular plate type brake which is mechanically actuated and its applied force is controlled using springs in accordance to:

Where, F=Braking force applied

$$
\mathrm{F}=\mathrm{Kx}
$$

$\mathrm{K}=$ Spring stiffness

$\mathrm{x}=$ displacement, (i.e., compression of the spring)

Through the years many different systems have been used for unwind braking, A system based on using a brake is one where a device imparts a drag force on the unwind shaft to allow the web tension force to be developed. we will consider brakes that are either pneumatic or electric in nature. In these cases either a pneumatic pressure or electrical power is applied to the brake assembly to generate the required torque. The resultant braking force is regulated by adjusting the pneumatic pressure or electric power which varies the drag that is applied to the unwind roll and therefore varies the tension.

The basic step for designing the brake is the calculation of the braking torque required. And once we have the braking torque we need to find out the braking force from this torque using:

Where: $\mathrm{T}=$ Braking torque

$$
\mathrm{T}=\mathrm{Fr}
$$

$\mathrm{F}=$ Braking force required

$\mathrm{r}=$ Radius of the shaft

The plate type brake we use is mounted on the frame and is applied on the fixed plate. The force applied by the disc brake is calculated by,

Where, $\mathrm{F}=$ Acting force

$$
\mathrm{F}=\mu \mathrm{AR}
$$

$\mu=$ Coefficient of friction

$\mathrm{R}=$ Reaction force

$\mathrm{A}=$ Surface area of the disc

Where, $\mathrm{A}=2 \pi \mathrm{r}$

$$
\text { and, } \quad r=\frac{1}{3} * \frac{r 1^{3}-r 2^{3}}{r 1^{2}-r 2^{2}}
$$

here, $\mathrm{r} 1=$ outer radius of the roll r2=inner radius of the roll

\subsection{Selection of motor speed:}

Selection of a suitable motor speed in order to suit our application is a very crucial part of the whole machine. As per the machines requirement there has to be a constant linear velocity of tissue feed but the diameter of the tissue roll keeps decreasing constantly thus we use the accumulator mechanism as stated above. Now a question arises that as the diameter of the tissue roll keeps decreasing continuously, It's angular velocity too keeps changing thus changing the linear velocity of roll feed in accordance to:

$$
\mathrm{V}=\pi \mathrm{DN} / 60
$$

Where: V=linear velocity

$\mathrm{D}=$ Diameter of the roll

$\mathrm{N}=$ R.P.M of the roll

As our objective is to keep the linear velocity constant we find the required r.p.m from the above equation. Here the linear velocity can be easily found out by considering the length $(\mathrm{L})$ of roll to be fed into the machine per minute and thus by using: 
$\mathrm{V}=\mathrm{L} / 60$

Now using this linear velocity we shall calculate the required r.p.m for the minimum diameter of the roll. We use this r.p.m since as the diameter decreases we need to increase the rate of feed in order to provide the same amount of tissue per minute, if we the r.p.m calculated by the larger diameter it will give us a lesser feed rate as the amount of tissue dispensed in one rotation equals the circumference of the roll thus it requires less rotations to achieve the same output as that for the roll with a smaller diameter.

IV.

CONCLUSIONS

All the mechanisms stated above have been designed keeping proper industrial standards in mind; this machine mainly consists of combinations of various small mechanisms which are actually used in the industry. The main criteria of designing was to keep it as simple as possible and using parts which would be easy to maintain and readily available locally. The whole mounting section has been divided into three parts namely the roll mount section, the accumulator and the machine intake section. There is no much information on the spool holder in this paper because, the spool holder need not be designed by us, there are various standards available in the market and all we have to do is select the one suitable for our application where as all the other components of the mounting section need to be designed specifically as per our required application.

\section{REFERENCES}

[1] Piotrowski, J.,Shaft Alignment Handbook, 2d ed., Marcel Dekker Inc., New York, 1995.

[2] GE Industrial Control Systems

[3] Flexo Presswise. Tension Control-Dirk Kroll-Webconvert Ltd

[4] The Mechanics Of Tension Control-By- Jeff Damour, Converter Accessory Corporatio], Wind Gap, PA USA4.

[5] Design Considerations in Unwind Tension Control Systems-by Bob Pasquale( New Era Converting Machinery)

[6] R, Butler R, H.J, Rump, A Web handling apparatus - US Patent-US3822838 A

[7] Y. Yasa, E. Sincar, B. T. Ertugrul, Erkan Mese, A multidisciplinary design approach for electromagnetic brakes. 